\section{CRITICAL MAPPINGS \\ OF RIEMANNIAN MANIFOLDS}

BY

DAVID D. BLEECKER

\begin{abstract}
We consider maps, from one Riemannian manifold to another, which are critical for all invariantly defined functionals on the space of maps. There are many such critical mappings, perhaps too numerous to suitably classify, although a characterization of sorts is provided. They are proven to have constant rank, with the image being a homogeneous minimal submanifold of the target manifold. Critical maps need not be Riemannian submersions onto their images. Also, there are homogeneous spaces for which the identity map is not critical. Many open problems remain.
\end{abstract}

1. Introduction. There are many invariantly defined functionals on the space of smooth mappings of one Riemannian manifold into another. One of the most widely studied is the energy functional (see [E2]). Maps that are critical for this functional are harmonic and possess nice properties. One can reasonably assert that the nicest (or most fundamental) maps in Riemannian geometry are those which are critical for every invariantly defined functional. We call such maps critical. The precise definition is found in $\S 1$. Some questions arise: Are there many examples of critical maps? How nice must they be? Can we hope to classify them?

We restrict ourselves to maps defined on a compact Riemannian manifold. We will exhibit many examples of critical maps, including the projection maps of Stiefel manifolds of $k$-frames in $\mathbf{F}^{n}(\mathbf{F}=\mathbf{R}, \mathbf{C}$, or $\mathbf{H})$ onto the Grassmann manifolds of $k$-planes in $\mathbf{F}^{n}$, homomorphisms of Lie groups with bi-invariant metrics, the Veronese surface in $S^{4}$, and many others (perhaps too numerous to suitably classify). We prove that the image of a critical map is a homogeneous minimal submanifold. Although we prove that critical maps have constant rank, they need not be Riemannian submersions onto their images in the sense of O'Neill [O]. (When they are, one can prove that the fibers must be minimal.) Among the counterexamples of $\$ 4$ is a left-invariant metric on $\mathrm{SU}(3)$ such that the identity map $\mathrm{SU}(3) \rightarrow \mathrm{SU}(3)$ is not critical.

This article is mainly an introduction to what will perhaps be perceived as

Received by the editors May 25, 1978.

AMS (MOS) subject classifications (1970). Primary 58D15, 58E99, 49F99, 53C20; Secondary $53 \mathrm{C} 40,53 \mathrm{C} 30$. 
a fundamental (as well as interesting) area of study. Thus, most of what follows consists of observations that may aid or motivate deeper investigations. Some possible directions for further research appear in $\$ 6$.

2. Notation, motivation, and definitions. Let $M$ and $N$ be $C^{\infty}$ manifolds with $M$ connected and compact. Let $\mathfrak{T}$ (resp., $\mathscr{T}$ ) be the space of all $C^{\infty}$ Riemannian metrics on $M$ (resp., $N$ ). Denote the space of diffeomorphisms of $M$ (resp., $N$ ) by $\mathscr{D}(M)$ (resp., $\mathscr{D}(N)$ ). There is a left action of $\mathscr{D}(M) \times$ $\mathscr{D}(N)$ on $\Re \times C^{\infty}(M, N) \times \Re$ given by $(\sigma, \eta) \cdot\left(g, f, g^{\prime}\right)=\left(\sigma^{-1^{*}} g\right.$, $\left.\eta \circ f \circ \sigma^{-1}, \eta^{-1^{*}} g^{\prime}\right)$. The triples $\left(g, f, g^{\prime}\right)$ and $(\sigma, \eta) \cdot\left(g, f, g^{\prime}\right)$ are geometrically equivalent, in the sense that we have a commutative diagram with the vertical maps being isometries:

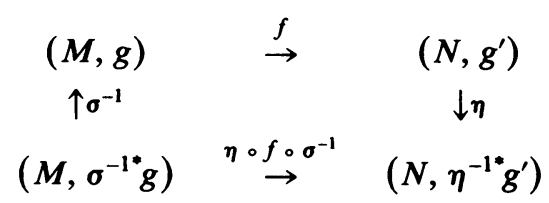

For $f \in C^{\infty}(M, N)$, we let $\mathcal{V}_{f}$ be the space of all vector fields along $f$. Now $\mathfrak{V}_{f}$ can be thought of as the tangent space to $C^{\infty}(M, N)$ at $f$, but we will not bother to make this precise. Given $g \in \mathfrak{N}$ and $g^{\prime} \in \Re$, we can define an inner product on $\mathcal{V}_{f}$ by

$$
\left\langle\phi_{1}, \phi_{2}\right\rangle=\int_{M} g^{\prime}\left(\phi_{1}(x), \phi_{2}(x)\right) \omega_{g}(x) .
$$

Let $S^{2}(M)$ (resp., $S^{2}(N)$ ) denote the space of symmetric covariant 2-tensor fields on $M$ (resp., $N$ ). We can define, for $g \in \mathfrak{N}$, an inner product on $S^{2}(M)$ by

$$
\langle s, t\rangle=\int_{M} s_{i j} t_{k p} g^{k i} g^{p j} \omega_{g} .
$$

For $g^{\prime} \in \mathfrak{R}$, we can define an inner product on the space $S^{2}(N)_{0}$ of compactly supported sections in $S^{2}(N)$ in the same way. Consider $S^{2}(M) \times$ $\mathfrak{V}_{f} \times S^{2}(N)_{0}$ to be the tangent space of $\Re \times C^{\infty}(M, N) \times \Re$ at $\left(g, f, g^{\prime}\right)$. Define an inner product on this tangent space by

$$
\left\langle\left(s_{1}, \phi_{1}, s_{1}^{\prime}\right),\left(s_{2}, \phi_{2}, s_{2}^{\prime}\right)\right\rangle=\left\langle s_{1}, s_{2}\right\rangle+\left\langle\phi_{1}, \phi_{2}\right\rangle+\left\langle s_{1}^{\prime}, s_{2}^{\prime}\right\rangle .
$$

Note that these inner products depend on the point $\left(g, f, g^{\prime}\right)$, but exhibiting the dependence is too cumbersome. It is a simple matter to check that for $(\sigma, \eta) \in \mathscr{D}(M) \times \mathscr{D}(N)$, we have $\left\|\left(\sigma^{-1^{*}} s, \eta_{*} \circ \phi \circ \sigma^{-1}, \eta^{-1^{*}} s^{\prime}\right)\right\|$ (at the point $\left.(\sigma, \eta) \cdot\left(g, f, g^{\prime}\right)\right)$ is equal to $\left\|\left(s, \phi, s^{\prime}\right)\right\|$ at $\left(g, f, g^{\prime}\right)$. Thus, we could say that $\mathscr{D}(M) \times \mathscr{D}(N)$ acts by isometries on the "Riemannian manifold" $\mathscr{T} \times$ $C^{\infty}(M, N) \times \Re$. The definitions to come are motivated by the well-known result: 
Proposition 2.1. Let $(M, g)$ be a (finite dimensional) Riemannian manifold, and let $G$ be a Lie subgroup of the group of isometries of $(M, g)$. Let $V \in T_{x} M$ be a tangent vector at $x \in M$. Then $V$ is the gradient at $x$ of some $G$-invariant $C^{\infty}$ function on $M$ iff $V$ is normal to the orbit of $G$ through $x$ and is fixed by the isotropy subgroup of $G$ at $x$.

The isotropy subgroup of $\mathscr{D}(M) \times \mathscr{D}(N)$ at $\left(g, f, g^{\prime}\right) \in \mathscr{T} \times C^{\infty}(M, N)$ $\times \mathcal{N}$ consists of all pairs of isometries $(\sigma, \eta) \in I_{g} \times I_{g^{\prime}}$ such that $\eta \circ f \circ \sigma^{-1}$ $=f$. We denote this subgroup by $I_{f}$, and let $I_{f}(M) \subset I_{g}$ and $I_{f}(N) \subset I_{g^{\prime}}$ be the projections of $I_{f}$ onto the first and second factors of $I_{g} \times I_{g^{\prime}}$. We now determine the space of vectors, based at $\left(g, f, g^{\prime}\right)$, normal to the orbit of $\left(g, f, g^{\prime}\right)$ under $\mathscr{D}(M) \times \mathscr{D}(N)$. Let $\sigma_{t} \in \mathscr{D}(M)$ and $\eta_{t} \in \mathscr{D}(N)$ be oneparameter groups of diffeomorphisms generated by vector fields $X$ on $M$ and $Y$ on $N$. A typical tangent vector to the orbit of $\left(g, f, g^{\prime}\right)$ is then

$$
\frac{d}{d t}\left[\left(\sigma_{t}, \eta_{t}\right) \cdot\left(g, f, g^{\prime}\right)\right]_{t=0}=\cdots=\left(-L_{X} g, Y \circ f-f_{*} X,-L_{Y} g^{\prime}\right)
$$

where $L$ denotes Lie differentiation. Thus, we say that $\left(s, \phi, s^{\prime}\right) \in S^{2}(M) \times$ $\mathfrak{V}_{f} \times S^{2}(N)_{0}$ is normal to the orbit of $\left(g, f, g^{\prime}\right)$ at $\left(g, f, g^{\prime}\right)$ if

$$
\left\langle\left(s, \phi, s^{\prime}\right),\left(-L_{X} g, Y \circ f-f_{*} X,-L_{Y} g^{\prime}\right)\right\rangle=0
$$

for all vector fields $X$ on $M$ and $Y$ on $N$. It is a standard fact (see [B2, p. 380]) that

$$
\left\langle s,-L_{X} g\right\rangle=2 \int_{M}[\operatorname{Div}(s)](X) \omega_{g}=2\left\langle\operatorname{Div}(s)^{\#}, X\right\rangle
$$

where the latter inner product is the ordinary $L^{2}$ inner product of vector fields on $(M, g)$. Using this fact and also that $s^{\prime}$ has compact support, we have that $\left(s, \phi, s^{\prime}\right)$ is normal iff

$$
\begin{array}{cc}
2\left\langle\operatorname{Div}(s)^{\sharp}, X\right\rangle-\left\langle\phi, f_{*} \circ X\right\rangle=0 & \text { for all } X \text { on } M, \\
\langle\phi, Y \circ f\rangle+2\left\langle\operatorname{Div}\left(s^{\prime}\right)^{\sharp}, Y\right\rangle=0 & \text { for all } Y \text { on } N .
\end{array}
$$

Condition (1) can be simplified. Define the adjoint of $f_{*}$ to be the vector bundle map $f_{*}^{t}: f^{*} T N \rightarrow T M$ defined pointwise by the formula $g\left(Z, f_{*}^{t} W\right)=$ $g^{\prime}\left(f_{*} Z, W\right)$. Since $\phi$ is a section of the bundle $f^{*} T N \rightarrow M$, we have that $f_{*}^{t} \phi$ is a vector field on $M$, and also $\left\langle\phi, f_{*} M\right\rangle=\left\langle f_{*}^{t} \phi, X\right\rangle$. Thus, we may replace (1) by the new condition

$$
2 \operatorname{Div}(s)^{\#}-f_{*}^{t} \phi=0 .
$$

Condition (2) presents some difficulty. The linear functional $Y \mapsto\langle\phi, Y \circ f\rangle$ 
is concentrated on $f(M)$ whereas, in general, the functional $Y \mapsto$ $2\left\langle\operatorname{Div}\left(s^{\prime}\right)^{\#}, Y\right\rangle$ is not. Thus, perhaps, in order for $\left(s, \phi, s^{\prime}\right)$ to be normal to the orbit, $s^{\prime}$ must be understood in a distributional sense and not as smooth section in $S^{2}(N)$. This situation, while probably not completely intractable, will be circumvented in this article, and perhaps considered at a later time.

We will avoid the difficulty by taking $\left(N, g^{\prime}\right)$ to be fixed. Let $\mathscr{D}(M) \times I_{g^{\prime}}$ act on the "submanifold" $\Re \times C^{\infty}(M, N) \times\left\{g^{\prime}\right\}$ in the same way as before. The vectors normal to the orbit of $\left(g, f, g^{\prime}\right)$ at $\left(g, f, g^{\prime}\right)$ under the action of $\mathscr{D}(M) \times I_{g^{\prime}}$ are then defined to be those $(s, \phi) \in S^{2}(M) \times \mathscr{V}_{f}$ such that the conditions (1)' and (2)' are met:

$$
\begin{gathered}
2 \operatorname{Div}(s)^{\#}-f_{*}^{t} \phi=0, \\
\langle\phi, Y \circ f\rangle=0 \text { for all Killing fields } Y \text { on }\left(N, g^{\prime}\right) .
\end{gathered}
$$

It is often the case that important theorems become definitions with the passage of time. Here we reverse this process by stating the next definition which hopefully will be a theorem in the near future. Thus, in analogy with Proposition 2.1, we have

DEFINITION 2.2. Let $\left(N, g^{\prime}\right)$ be a fixed Riemannian manifold. We say that $f$ : $(M, g) \rightarrow\left(N, g^{\prime}\right)$ is critical if for all pairs $(s, \phi) \in S^{2}(M) \times \mathfrak{V}_{f}$, which are normal to the orbit of $\left(g, f, g^{\prime}\right)$ (under the action of $\mathscr{D}(M) \times I_{g^{\prime}}$ on $\Re \times$ $\left.C^{\infty}(M, N) \times\left\{g^{\prime}\right\}\right)$ at $\left(g, f, g^{\prime}\right)$ and are left fixed by the isotropy subgroup $I_{f} \subset I_{g} \times I_{g^{\prime}}$, we have $\phi=0$.

In view of the previous definitions and observations we have made, we have the equivalent working definition of critical:

Definition 2.3. We say that $f:(M, g) \rightarrow\left(N, g^{\prime}\right)$ is critical if for all pairs $(s, \phi) \in S^{2}(M) \times \mathcal{V}_{f}$ satisfying $(\alpha),(\beta)$, and $(\gamma)$ below, we have $\phi=0$ :

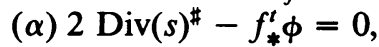

( $\beta)\langle\phi, Y \circ f\rangle=0$ for all Killing fields $Y$ on $\left(N, g^{\prime}\right)$,

$(\gamma) \eta_{*} \circ \phi \circ \sigma^{-1}=\phi$ and $\sigma_{*}^{-1} s=s$ for all $(\sigma, \eta) \in I_{f}$.

Note that, in strict analogy with Proposition 2.1, perhaps we should require $s=0$, in addition to $\phi=0$. If we did this, then no map $f$ could be critical because $(g, 0) \in S^{2}(M) \times \mathcal{V}_{f}$ satisfies $(\alpha),(\beta)$, and $(\gamma)$. If we add an additional requirement,

( $\delta) s \neq \lambda g$ for all nonzero $\lambda \in \mathbf{R}$,

then it is proved in [B3] that $(M, g)$ would have to be an isotropy irreducible homogeneous space if we require $s=0$. While this probably leads to a nice theory, we can prove quite a bit about critical maps without this additional restriction. Also, we make some comments about the sense in which Definition 2.2 is probably a theorem. What is really needed to "justify" Definition 2.2 is a slice theorem for the action of $\mathscr{D}(M) \times I_{g^{\prime}}$ on $\Re \times C^{\infty}(M, N) \times$ $\left\{g^{\prime}\right\}$. The author believes that there is such a theorem. One crucial property 
needed is that the normal bundle to the orbit consist of vectors that make up the kernel of a differential operator whose adjoint has an injective symbol. Then one can probably proceed, as in [E1], to produce a slice. For the problem at hand, the differential operator is $P: S^{2}(M) \oplus \Gamma\left(f^{*} T N\right) \rightarrow$ $\Gamma(T M)$, given by $P(s, \phi)=2 \operatorname{Div}(s)^{\#}-f_{*}^{t} \phi$. Vectors normal to the orbit are then defined by $P(s, \phi)=0$ (i.e., $(\alpha)$ ), subject to the innocent side condition $(\beta)$. Note that $P$ is a first order differential operator with principal part being $2 \operatorname{Div}(\cdot)^{\sharp}$. The adjoint of $2 \operatorname{Div}(\cdot)^{\#}$ is $-L_{(\cdot)} g$ which is known to have an injective symbol [B2].

As a useful check on Definition 2.3, we prove a theorem. Consider a functional $F$ : $\mathfrak{N} \times C^{\infty}(M, N) \times\left\{g^{\prime}\right\} \rightarrow \mathbf{R}$ which is invariantly defined, in the sense that $F$ is constant on the orbits of the action of $\mathscr{D}(M) \times I_{g^{\prime}}$. Suppose also that $F$ is smooth, in the sense that, for each $\left(g, f, g^{\prime}\right) \in \Re \times$ $C^{\infty}(M, N) \times\left\{g^{\prime}\right\}$, we have a pair $\left(D_{1} F, D_{2} F\right) \in S^{2}(M) \times \mathscr{V}_{f}$ such that, for all $(s, \phi) \in S^{2}(M) \times \mathcal{V}_{f}$ (and where $\left(\exp _{f} t \phi\right)(x) \equiv \exp _{f(x)}(t \phi(x))$ ), we have (at $t=0$ )

$$
\frac{d}{d t} F\left(g+t s, \exp _{f}(t \phi)\right)=\left\langle D_{1} F, s\right\rangle+\left\langle D_{2} F, \phi\right\rangle
$$

THEOREM 2.4. Under the above assumptions, $\left(D_{1} F, D_{2} F\right)$ meets conditions $(\alpha),(\beta)$, and $(\gamma)$ of Definition 2.3.

Proof. Let $X$ be a vector field on $M$ with one-parameter group $\sigma_{t}$, and let $Y$ be a Killing vector field on $\left(N, g^{\prime}\right)$ with one-parameter group of isometries $\eta_{t}$. Then, at $t=0$, we have

$$
\begin{aligned}
0 & =\frac{d}{d t} F\left(\sigma_{t}^{-1^{*}} g, \eta_{t} \circ f \circ \sigma_{t}^{-1}\right) \\
& =\frac{d}{d t} F\left(g-t L_{X} g, \exp _{f}\left(t\left(Y \circ f-f_{*} X\right)\right)\right) \\
& =\left\langle D_{1} F,-L_{X} g\right\rangle+\left\langle D_{2} F, Y \circ f-f_{*} X\right\rangle \\
& =\left\langle 2 \operatorname{Div}\left(D_{1} F\right)^{\#}-f_{*}^{t} D_{2} F, X\right\rangle+\left\langle D_{2} F, Y \circ f\right\rangle .
\end{aligned}
$$

Thus, we have conditions $(\alpha)$ and $(\beta)$. As for $(\gamma)$, suppose $(\sigma, \eta) \in I_{f}$, and let $(\sigma, \eta)$ denote also the map

$$
\left(g, f, g^{\prime}\right) \mapsto(\sigma, \eta) \cdot\left(g, f, g^{\prime}\right)
$$

of $\mathfrak{T} \times C^{\infty}(M, N) \times\left\{g^{\prime}\right\}$ into itself. Set $D F \equiv\left(D_{1} F, D_{2} F\right)$. Then, using the fact $F=F \circ(\sigma, \eta)$ we have, for all $(s, \phi) \in S^{2}(M) \times \mathcal{V}_{f}$, at $t=0$, 


$$
\begin{aligned}
\left\langle D_{1} F, s\right\rangle+\left\langle D_{2} F, \phi\right\rangle & =\langle D F,(s, \phi)\rangle \\
& =\langle D(F \circ(\sigma, \eta)),(s, \phi)\rangle \\
& =\frac{d}{d t}\left[(F \circ(\sigma, \eta))\left(g+t s, \exp _{f} t \phi\right)\right] \\
& =\frac{d}{d t} F\left(\sigma^{-1^{*}}(g+t s), \eta \circ \exp _{f \circ \sigma^{-1}}\left(t \phi \circ \sigma^{-1}\right)\right) \\
& =\frac{d}{d t} F\left(g+t \sigma^{-1^{*}} s, \exp _{\eta \circ f \circ \sigma^{-1}}\left(t \eta_{*} \circ \phi \circ \sigma^{-1}\right)\right) \\
& =\frac{d}{d t} F\left(g+t \sigma^{-1^{*}} s, \exp _{f}\left(t \eta_{*} \circ \phi \circ \sigma^{-1}\right)\right) \\
& =\left\langle D_{1} F, \sigma^{-1^{*}} s\right\rangle+\left\langle D_{2} F, \eta_{*} \circ \phi \circ \sigma^{-1}\right\rangle \\
& =\left\langle\sigma^{*} D_{1} F, s\right\rangle+\left\langle\eta_{*}^{-1} \circ D_{2} F \circ \sigma, \phi\right\rangle .
\end{aligned}
$$

Thus, $\sigma^{*} D_{1} F=D_{1} F$ and $\eta_{*}^{-1} \circ D_{2} F \circ \sigma=D_{2} F$, and so we have $(\gamma)$.

COROllary 2.5. If $f:(M, g) \rightarrow(N, g)$ is critical, then $f$ is harmonic.

Proof. Consider the energy functional $F$ : $\mathfrak{T} \times C^{\infty}(M, N) \times\left\{g^{\prime}\right\} \rightarrow \mathbf{R}$ given by

$$
F\left(g, f, g^{\prime}\right)=\frac{1}{2}\left\langle g, f^{*} g^{\prime}\right\rangle .
$$

By a computation in [E2, p. 116], it is shown that $D_{2} F=-\Delta f$ where $\Delta f$ denotes the Laplacian of $f$ (i.e. the trace of the covariant derivative of $f_{*}$ in the bundle $\left.\operatorname{Hom}\left(T M, f^{*} T N\right)\right)$. By a computation,

$$
2 D_{1} F=\frac{1}{2} \operatorname{trace}\left(f^{*} g^{\prime}\right) g-f^{*} g^{\prime}
$$

where trace $\left(f^{*} g^{\prime}\right)=\left(f^{*} g^{\prime}\right)_{i j} g^{i j}$. As $F$ is certainly constant on the orbits of the action by $\mathscr{D}(M) \times I_{g^{\prime}}$, Theorem 2.5 applies, and we have $-\Delta f=D_{2} F=0$, since $f:(M, g) \rightarrow\left(N, g^{\prime}\right)$ is assumed critical.

There is a stronger notion of critical which is obtained if we drop ( $\alpha)$ and $(\beta)$ in Definition 2.3, and require that $(\gamma)$ alone implies $\phi=0$. In this case, we say that $f:(M, g) \rightarrow\left(N, g^{\prime}\right)$ is hypercritical. Clearly, hypercritical implies critical. In $\S 3$, we prove that if $I_{f}(M)$ does not act transitively on $M$, then hypercritical and critical are equivalent notions. Whether critical implies hypercritical, in general, is yet unknown to the author.

3. Some basic results.

Proposition 3.1. Let $(M, g)$ be a compact Riemannian manifold. If $X$ is any vector field on $M$, then $X=\operatorname{Div}(s)^{\sharp}$ for some $s \in S^{2}(M)$ iff $\langle X, Y\rangle=0$ for all Killing vector fields $Y$ on $(M, g)$. 
Proof. Recall the formula $2\left\langle\operatorname{Div}(s)^{\sharp}, Y\right\rangle=\left\langle s,-L_{Y} g\right\rangle$. From this we see that if $X=\operatorname{Div}(s)^{\sharp}$ and $Y$ is Killing, then $\langle X, Y\rangle=0$. Conversely, consider the differential operator $L_{(\cdot)} g: \Gamma(T M) \rightarrow S^{2}(M)$. The symbol of this operator is injective [B2]. Since the adjoint of $L_{(\cdot)} g$ is $-2 \operatorname{Div}(\cdot)^{\#}$, we have that

$$
A \equiv-2 \operatorname{Div}(\cdot)^{\#} \circ L_{(\cdot)} g: \Gamma(T M) \rightarrow \Gamma(T M)
$$

is elliptic and selfadjoint. From the theory of elliptic operators, we have the orthogonal direct sum $\Gamma(T M)=\operatorname{ker} A \oplus \operatorname{im} A$. If $X$ is Killing, then certainly $A X=0$, and conversely, if $A X=0$, then

$$
0=\langle A X, X\rangle=\left\langle-2 \operatorname{Div}\left(L_{X} g\right)^{\#}, X\right\rangle=\left\langle L_{X} g, L_{X} g\right\rangle,
$$

and so $X$ is Killing. Thus, $\operatorname{ker} A$ coincides with the space of Killing vector fields. Now every field orthogonal to $\operatorname{ker} A$ is in im $A$ which consists only of fields which are divergences ${ }^{\#}$ (by definition of $A$ ).

Corollary 3.2. The map $f:(M, g) \rightarrow\left(N, g^{\prime}\right)$ is critical iff for all $\phi \in \mathfrak{V}_{f}$ satisfying $(\alpha)^{\prime},(\beta)^{\prime},(\gamma)^{\prime}$ below, we have $\phi=0$.

$(\alpha)^{\prime}\left\langle f_{*}^{t} \phi, X\right\rangle=0$ for all Killing fields $X$ on $(M, g)$,

$(\beta)^{\prime}\langle\phi, Y \circ f\rangle=0$ for all Killing fields $Y$ on $\left(N, g^{\prime}\right)$,

$(\gamma)^{\prime} \eta_{*} \circ \phi \circ \sigma^{-1}=\phi$ for all $(\sigma, \eta) \in I_{f}$.

Proof. We know that $(\alpha)^{\prime}$ implies that $f_{*}^{t} \phi=2 \operatorname{Div}(s)^{\#}$ for some $s \in$ $S^{2}(M)$, but in compliance with $(\gamma)$ of Definition 2.3, we need to prove that $s$ can be chosen to be invariant under $I_{f}(M)$. First we prove that $f_{*}^{t} \phi$ is invariant under $I_{f}(M)$, if $(\gamma)^{\prime}$ holds. Indeed, for every vector $V_{x} \in T_{x} M$, we have

$$
\begin{aligned}
g\left(\left(\sigma_{*} f_{*}^{t} \phi\right)_{x}, V_{x}\right) & =g\left(\sigma_{*} f_{*}^{t} \phi\left(\sigma^{-1} x\right), V_{x}\right) \\
& =g\left(f_{*}^{t} \phi\left(\sigma^{-1} x\right), \sigma_{*}^{-1} V_{x}\right) \\
& =g^{\prime}\left(\phi\left(\sigma^{-1}(x)\right), f_{*} \sigma_{*}^{-1} V_{x}\right) \\
& =g^{\prime}\left(\eta_{*} \phi\left(\sigma^{-1}(x)\right), \eta_{*} f_{*} \sigma_{*}^{-1} V_{x}\right) \\
& =g^{\prime}\left(\phi(x), f_{*} V_{x}\right)=g\left(f_{*}^{t} \phi(x), V_{x}\right) .
\end{aligned}
$$

Thus, $\sigma_{*} f_{*}^{t} \phi=f_{*}^{t} \phi$ for all $\sigma \in I_{f}(M)$. Let $\mu$ be normalized Haar measure on the compact Lie group $I_{f}(M)$, and define

$$
\bar{s}_{x}=\int_{I_{f}(M)}\left(\sigma^{*} s\right)_{x} d \mu(\sigma) .
$$

Then $\bar{s}$ is invariant under $I_{f}(M)$. Indeed this averaging operation extends to the entire tensor algebra and commutes with all the usual differential operators such as Div, because of theorems regarding differentiation under integrals and the fact that $I_{f}(M) \subset I_{g}$. Thus, we have 


$$
f_{*}^{t} \phi=\overline{f_{*}^{t} \phi}=\overline{2 \operatorname{Div}(s)^{\sharp}}=2 \operatorname{Div}(\bar{s})^{\sharp},
$$

as required.

Recall that a map is hypercritical if $(\gamma)$ (or equivalently $\left.(\gamma)^{\prime}\right)$ alone implies $\phi=0$.

THEOREM 3.3. If $I_{f}(M)$ is not transitive on $M$, then $f$ is hypercritical iff $f$ is critical.

Proof. Under any circumstances $f$ hypercritical implies $f$ critical. Thus, assume $f$ is not hypercritical. Then there is a $\phi \in \mathscr{V}_{f}, \phi \neq 0$, such that $\eta_{*} \circ \phi \circ \sigma^{-1}=\phi$ for all $(\sigma, \eta) \in I_{f}$. Let $S=\{x \in M: \phi(x) \neq 0\}$, and let $\mathscr{F}$ be the vector space of all $C^{\infty}$ functions on $M$ invariant under $I_{f}(M)$ and with supports in $S$. Since $I_{f}(M)$ is assumed to be not transitive, $\mathscr{F}$ is infinite dimensional. If $h \in \mathcal{F}$, then we may define $h \phi \in \mathcal{V}_{f}$ by $(h \phi)(x)=h(x) \phi(x)$. We easily find that $h \phi$ satisfies $(\gamma)^{\prime}$. Let $X_{1}, \ldots, X_{k}$ be a basis for the space of Killing vector fields on $(M, g)$, and $Y_{1}, \ldots, Y_{j}$ be such a basis for $\left(N, g^{\prime}\right)$. Define linear functionals $\alpha_{1}, \ldots, \alpha_{k}$ and $\beta_{1}, \ldots, \beta_{j}$ on $\mathscr{F}$ by $\alpha_{i}(h)=$ $\left\langle f_{*}^{t} h \phi, X_{i}\right\rangle$ and $\beta_{i}(h)=\left\langle h \phi, Y_{i}\right\rangle$. As $\mathscr{F}$ is infinite dimensional, the subspace of all $h \in \mathcal{F}$ annihilated by all the functionals $\alpha_{1}, \ldots, \alpha_{k}, \beta_{1}, \ldots, \beta_{j}$ is still infinite dimensional. Let $h \neq 0$ be in this subspace. Then $h \phi \neq 0$, since the support of $h$ is in $S$. By construction, $h \phi$ satisfies $(\alpha)^{\prime},(\beta)^{\prime}$, and $(\gamma)^{\prime}$. Thus, $f$ is not critical.

THEOREM 3.4. If $f:(M, g) \rightarrow\left(N, g^{\prime}\right)$ is critical, then $I_{f}(N)$ acts transitively on the set $f(M) \subset N$.

Proof. If $I_{f}(M)$ acts transitively on $M$, then the result is clear. Thus, suppose $I_{f}(M)$ does not act transitively. By Theorem 3.3, we then know that $f$ is hypercritical. Assume that $I_{f}(N)$ does not act transitively on $f(M)$. Then it is certainly possible to find $h \in C^{\infty}(N)$ such that $h$ is invariant under $I_{f}(N)$ and is not constant on $f(M)$. Define $\phi \in \mathcal{V}_{f}$ by $\phi(x)=\nabla h(f(x))$. Since $h$ is not constant on $f(M), \phi \neq 0$. It is easy to prove that $\phi$ satisfies $(\gamma)^{\prime}$. This contradicts $f$ being hypercritical.

Corollary 3.5. If $f:(M, g) \rightarrow\left(N, g^{\prime}\right)$ is critical, then $f(M)$ is an orbit of the compact subgroup $I_{f}(N)$ of $I_{g^{\prime}}$. Thus, $f(M)$ is an imbedded submanifold which is a homogeneous space relative to the induced metric.

Proof. By Theorem 3.4, it suffices to prove that $f(M)$ is preserved by $I_{f}(N)$, but that is clear.

Corollary 3.6. If $f:(M, g) \rightarrow\left(N, g^{\prime}\right)$ is critical, then $f_{*}$ has constant rank $=\operatorname{dim} f(M)$. 
Proof. Since $f(M)$ is a manifold, we may apply Sard's theorem to the smooth map $f: M \rightarrow f(M)$. We deduce that the critical values have measure 0 in $f(M)$. We also know that if there is one critical value in $f(M)$, then all points of $f(M)$ are critical values, since $I_{f}(N)$ is transitive. Thus, $f: M \rightarrow f(M)$ must be regular everywhere, whence $f: M \rightarrow N$ has constant rank= $\operatorname{dim} f(M)$.

THEOREM 3.7. If $f:(M, g) \rightarrow\left(N, g^{\prime}\right)$ is critical, then $f(M)$ is a minimal submanifold of $\left(N, g^{\prime}\right)$.

Proof. Let $H$ be the mean curvature vector field on $f(M)$. Define $\phi \in \mathcal{V}_{f}$ by $\phi(x)=H(f(x))$. For $(\sigma, \eta) \in I_{f}$,

$$
\begin{aligned}
\eta_{*} \phi\left(\sigma^{-1}(x)\right) & =\eta_{*} H\left(f\left(\sigma^{-1} x\right)\right)=H\left(\eta \circ f \circ \sigma^{-1}(x)\right) \\
& =H(f(x))=\phi(x) .
\end{aligned}
$$

Thus, $\phi$ satisfies $(\gamma)^{\prime}$. Now $f_{*}^{t} \phi=0$, since $H \perp f(M)$, and so $(\alpha)^{\prime}$ is met. Let $Y$ be a Killing vector field on $\left(N, g^{\prime}\right)$ with one-parameter group of isometries $\eta_{t}$. Then the volume of $\eta_{t}(f(M))$ is constant, and so

$$
0=\frac{d}{d t}\left(\text { volume of } \eta_{t}(f(M))\right)=-\langle Y, H\rangle .
$$

It follows that $\langle Y \circ f, \phi\rangle=0$. This requires a little work, because $\langle Y, H\rangle$ is an integral over $f(M)$ while $\langle Y \circ f, \phi\rangle$ is the integral, of the pullback of the function $g^{\prime}(Y, H)$, over $M$. However, since $I_{f}(N)$ is transitive on $f(M)$, it is easy to see that there is a constant $c>0$ such that for all $h \in C^{\infty}(f(M))$,

$$
\int_{M} f^{*} h \omega_{g}=c \int_{f(M)} h\left(\omega_{g^{\prime}} \mid f(M)\right) .
$$

Thus, $\phi$ meets $(\beta)^{\prime}$ as well, and $f$ critical implies $\phi=0$, whence $H=0$.

The most satisfying characterization of critical maps is Theorem 3.10. First we state:

Definition 3.8. Let $\phi \in \mathscr{V}_{f}$ be of the form $\phi=f_{*}(X)+Y \circ f$, where $X$ is Killing on $(M, g)$ and $Y$ is Killing on $\left(N, g^{\prime}\right)$. Such fields are called infinitesimal isodeformations of $f$ (inf. isodefs.). (This is because $\phi$ is then $\left.(d / d t)\left(\eta_{t} f \sigma_{t}\right)\right|_{t=0}$ for the one-parameter groups of isometries $\sigma_{t}$ and $\eta_{t}$ of $X$ and $Y$, respectively.)

DEFINITION 3.9. Let $\rho: I_{f} \rightarrow \operatorname{GL}\left(\mathcal{V}_{f}\right)$ be the representation $\rho((\sigma, \eta))(\phi)=$ $\eta_{*} \circ \phi \circ \sigma^{-1}$. We call $\phi I_{f}$-invariant if $\rho((\sigma, \eta)) \phi=\phi$. (This is equivalent to condition $(\gamma)^{\prime}$.)

THEOREM 3.10. $f:(M, g) \rightarrow\left(N, g^{\prime}\right)$ is critical iff every $I_{f}$-invariant field along $f$ is an infinitesimal isodeformation.

Proof. Let $\mathcal{K}$ be the vector space of inf. isodefs. of $f$, and let $\mathcal{f}$ be the vector space of $I_{f}$-invariant fields in $\mathfrak{V}_{f}$. Let $\phi \in \mathfrak{V}_{f}$. Observe that $\left(\alpha^{\prime}\right)$ and 
( $\left.\beta^{\prime}\right)$ together are equivalent to $\langle\phi, \mathscr{K}\rangle=0$, while $\left(\gamma^{\prime}\right)$ means $\phi \in \mathcal{G}$. Thus, if $\mathcal{G} \subset \mathcal{K}$, then $(\alpha)^{\prime},(\beta)^{\prime},(\gamma)^{\prime}$ imply $\langle\phi, \phi\rangle=0$ or $\phi \equiv 0$, whence $f$ is critical. Conversely, assume that $f$ is critical. If $I_{f}(M)$ does not act transitively on $M$, then $g=0$, since $f$ would be hypercritical by Theorem 3.3. In this case, we are done. If $I_{f}(M)$ acts transitively, then $g$ is finite dimensional, since each $\phi \in \mathcal{G}$ is then determined by the value of $\phi$ at a point $x\left(\phi(\sigma(x))=\eta_{*} \phi(x)\right.$ for $\left.(\sigma, \eta) \in I_{f}\right)$. Observe that $\mathcal{K}$ is an invariant subspace relative to $\rho$ of 3.9. Indeed, for $(\sigma, \eta) \in I_{f}$ and $f_{*} X+Y \circ f \in \mathcal{K}$, we compute

$$
\begin{aligned}
\eta_{*} \circ\left(f_{*} X+Y \circ f\right) \circ \sigma^{-1} & =\cdots \\
& =f_{*}\left(\sigma_{*} \circ X \circ \sigma^{-1}\right)+\left(\eta_{*} \circ Y \circ \eta^{-1}\right) \circ f \in \mathscr{K} .
\end{aligned}
$$

Thus, $\mathscr{f}+\mathcal{K}$ (not necessarily direct sum) is an invariant subspace of $\rho$. We have remarked before that $\rho$ is orthogonal relative to $\langle$,$\rangle on \mathfrak{V}_{f}$, and so the restriction $\rho_{0}: I_{f} \rightarrow O(\mathcal{F}+\mathcal{K})$ is an orthogonal finite dimensional representation of $I_{f}$. Thus, we may write

$$
\mathcal{G}+\mathcal{K}=\mathfrak{L}_{1} \oplus \cdots \oplus \mathcal{L}_{p} \oplus \mathcal{K}_{1} \oplus \cdots \oplus \mathcal{K}_{q},
$$

an orthogonal direct sum of irreducible invariant subspaces, where $\mathcal{K}=\mathcal{K}_{1}$ $\oplus \cdots \oplus \mathcal{K}_{q}$. Note that $g$ is the direct sum of the trivial one-dimensional summands. Since we are assuming $f$ is critical, and as the direct sum is orthogonal, none of these trivial summands is among the $\mathfrak{E}_{i}$. Hence $\mathscr{f} \subset \mathscr{K}$.

THEOREM 3.11. If $f:(M, g) \rightarrow\left(N, g^{\prime}\right)$ is hypercritical, then the fibers $f^{-1}(y)$ are minimal submanifolds of $(M, g)$.

Proof. Corollary 3.6 implies that the fibers are submanifolds of $M$. Let $H$ be the vector field on $M$ defined by $H(x)=$ mean curvature vector of $f^{-1}(f(x))$ at $x$. Define $\phi \in \mathfrak{V}_{f}$ by requiring that $\phi(x) \in T_{f(x)} f(M)$ satisfy

$$
g(X, H(x))=g^{\prime}\left(f_{*}(X), \phi(x)\right)
$$

for all $X \in T_{x} M$. Note that $\phi(x)$ exists, since $H \perp \operatorname{ker} f_{*}$; and $\phi(x)$ is unique, since $f_{*}$ maps $T_{x} M$ onto $T_{f(x)} f(M) \ni \phi(x)$. Observe that $H=f_{*}^{t} \phi$. For $(\sigma, \eta) \in I_{f}$ and $X \in T_{x} M$,

$$
\begin{aligned}
g^{\prime}\left(\eta_{*} \phi\left(\sigma^{-1}(x)\right), f_{*} X\right) & =g^{\prime}\left(\phi\left(\sigma^{-1}(x)\right), \eta_{*}^{-1} f_{*} X\right) \\
& =g^{\prime}\left(\phi\left(\sigma^{-1}(x)\right), f_{*} \sigma_{*}^{-1} X\right) \\
& =g\left(H\left(\sigma^{-1}(x)\right), \sigma_{*}^{-1} X\right) \\
& =(\text { since } \sigma \text { is a fiber-preserving isometry }) \ldots \\
& =g\left(\sigma_{*}^{-1} H(x), \sigma_{*}^{-1} X\right)=g(H(x), X) \\
& =g^{\prime}\left(\phi(x), f_{*} X\right) .
\end{aligned}
$$


Thus, $\eta_{*} \phi\left(\sigma^{-1}(x)\right)=\phi(x)$, and so $\phi$ is $I_{f}$-invariant. As $f$ is assumed to be hypercritical, $\phi=0$, and so $H=0$.

Note that $f_{*}^{t} \phi=H$ satisfies $(\alpha)^{\prime}$ of Corollary 3.2, using the argument in Theorem 3.7. However, I do not know whether $(\beta)^{\prime}$ is met in general. Thus, the question as to whether critical maps always have minimal fibers is open. A partial answer is given by 3.16. If critical implies hypercritical, then the whole question is resolved by Theorem 3.11 .

Definition 3.12. Let $f:(M, g) \rightarrow\left(N, g^{\prime}\right)$ be called a Riemannian mapping if, for all $x \in M$ and $X, Y \in\left(\operatorname{ker} f_{*}\right)_{x}^{\perp} \subset T_{x} M$, we have $g^{\prime}\left(f_{*} X, f_{*} Y\right)=$ $g(X, Y)$.

Certainly, if $f$ is Riemannian, then $f$ must have constant rank. Conversely, if $f$ has constant rank, there is a canonically constructed metric $g^{\prime \prime}$ on $M$ such that $f:\left(M, g^{\prime \prime}\right) \rightarrow\left(N, g^{\prime}\right)$ is Riemannian. Letting $\pi_{H}$ and $\pi_{V}$ be the projections of $T M$ onto the subbundles $\left(\operatorname{ker} f_{*}\right)^{\perp}$ (relative to $g$ ) and $\operatorname{ker} f_{*}$, we define

$$
g^{\prime \prime}(X, Y)=g\left(\pi_{V} X, \pi_{V} Y\right)+g^{\prime}\left(f_{*} \pi_{H} X, f_{*} \pi_{H} Y\right)
$$

Then $f:\left(M, g^{\prime \prime}\right) \rightarrow\left(N, g^{\prime}\right)$ is Riemannian. Let

$$
\bar{I}_{f}=\left\{(\sigma, \eta) \in I_{g^{\prime \prime}} \times I_{g^{\prime}}: \eta \circ f \circ \sigma^{-1}=f\right\}
$$

and let $I_{f} \subset I_{g} \times I_{g^{\prime}}$ be as before.

THEOREM 3.13. $I_{f} \subset \bar{I}_{f}$.

Proof. Let $(\sigma, \eta) \in I_{f}$. Then $\sigma^{*} g=g, \eta \circ f \circ \sigma^{-1}=f$, and $\eta^{*} g^{\prime}=g^{\prime}$. We need only prove that $\sigma^{*} g^{\prime \prime}=g^{\prime \prime}$. Note that, for $X \in \operatorname{ker} f_{* x}$, we have $f_{* \sigma(x)}\left(\sigma_{*} X\right)=\eta_{*}\left(f_{* x} X\right)=0$. Thus, $\sigma_{*}: T M \rightarrow T M$ preserves the subbundle $\operatorname{ker} f_{*}$, and hence also $\left(\operatorname{ker} f_{*}\right)^{\perp}\left(\right.$ since $\left.\sigma \in I_{g}\right)$. Observe that $\left(\operatorname{ker} f_{*}\right)^{\perp}$ relative to $g$ is $\left(\operatorname{ker} f_{*}\right)^{\perp}$ relative to $g^{\prime \prime}$. By a straightforward computation using the definition of $g^{\prime \prime}$ and the fact $(\sigma, \eta) \in I_{f}$, we can prove that $\sigma^{*} g^{\prime \prime}(X, Y)=$ $g^{\prime \prime}(X, Y)$ in the three cases where both $X, Y$ are horizontal, vertical, or one horizontal and the other vertical.

COROllary 3.14. If $f:(M, g) \rightarrow\left(N, g^{\prime}\right)$ is hypercritical, then $f:\left(M, g^{\prime \prime}\right) \rightarrow$ $\left(N, g^{\prime}\right)$ is hypercritical.

Proof. Simply use Theorem 3.13 and the definition of hypercritical.

There are difficulties with Corollary 3.14 in the critical case, since the change of metric might decrease the space of infinitesimal isodeformations of $f$ (see Theorem 3.10).

TheOREM 3.15. Let $f:(M, g) \rightarrow\left(N, g^{\prime}\right)$ be a Riemannian mapping. Then $f$ is harmonic iff the fibers are minimal and the image of $f$ is an immersed minimal submanifold. 
Proof. Recall that the Laplacian $\Delta f$ is the trace of the tensor $\Omega \in$ $\Gamma\left(\operatorname{Hom}\left(T M \times T M, f^{*} T N\right)\right)$ defined by

$$
\Omega_{x}(X, Y)=\nabla_{f_{*}(X)}^{\prime} f_{*}(\bar{Y})-f_{*}\left(\nabla_{X} \bar{Y}\right)
$$

where $\nabla$ and $\nabla^{\prime}$ are the Riemannian connections of $(M, g)$ and $\left(N, g^{\prime}\right)$, and $\bar{Y}$ is an extension of $Y \in T_{x} M$ such that $f_{*}(\bar{Y})$ is well defined. Now $\Omega$ is symmetric, and so is independent of the extension $\bar{Y}$. If $Z$ and $W$ are vector fields tangent to $f(M)$, and $\tilde{Z}$ and $\tilde{W}$ are horizontal lifts of $Z$ and $W$ to $M$, then (since $f$ is Riemannian) we find that $\left(\nabla_{Z}^{\prime} W\right)^{T}=f_{*}\left(\nabla_{\tilde{Z}} \tilde{W}\right)$ where $T$ denotes projection of $T N \mid f(M)$ onto $T f(M)$. Let $E_{1}, \ldots, E_{p}$ be orthonormal fields that frame $f(M)$ in a small neighborhod of $f(x)$ in $f(M)$, where $p$ is the constant rank of $f$. Let $\tilde{E}_{1}, \ldots, \tilde{E}_{p}$ denote the horizontal lifts of $E_{1}, \ldots, E_{p}$. Since $f$ is Riemannian, these lifts are orthonormal. Complete $\tilde{E}_{1}, \ldots, \tilde{E}_{p}$ to an orthonormal frame $\tilde{E}_{1}, \ldots, \tilde{E}_{p}, F_{1}, \ldots, F_{q}$ on a neighborhood of $x$. Then

$$
\begin{aligned}
\Delta f_{x} & =\sum_{1}^{p} \Omega\left(\tilde{E}_{i}, \tilde{E}_{i}\right)+\sum_{1}^{q} \Omega\left(F_{j}, F_{j}\right) \\
& =\sum_{1}^{p}\left(\nabla_{E_{i}}^{\prime} E_{i}-f_{*}\left(\nabla_{\tilde{E}_{i}} \tilde{E}_{i}\right)\right)+\sum_{1}^{q}\left(0-f_{*}\left(\nabla_{F_{j}} F_{j}\right)\right) \\
& =\sum_{1}^{p}\left(\nabla_{E_{i}}^{\prime} E_{i}\right)^{N}-f_{*}\left(H_{x}\right) \\
& =H_{f(x)}^{\prime}-f_{*}\left(H_{x}\right)
\end{aligned}
$$

where $H^{\prime}$ is the mean curvature vector of $f(M)$ in $N$ and $H$ is that of the fiber. Thus, $\Delta f=0$ iff $H=0$ and $H^{\prime}=0$.

Corollary 3.16. If $f:\left(M, g^{\prime}\right) \rightarrow\left(N, g^{\prime}\right)$ is a critical Riemannian mapping, then the fibers of $f$ are minimal.

Proof. Recall Corollary 2.5.

4. Examples and counterexamples. Many standard examples of critical maps arise from the following trivial result.

Theorem 4.1. Let $\left(N, g^{\prime}\right)$ be a symmetric space, and let $f:(M, g) \rightarrow\left(N, g^{\prime}\right)$ have the property that, for any $x \in M$, there is $a \sigma \in I_{f}(M)$ such that $\sigma(x)=x$ and $(\sigma, \eta) \in I_{f}$, where $\eta$ is the geodesic symmetry of $\left(N, g^{\prime}\right)$ at $f(x)$. Then $f$ is hypercritical.

Proof. Let $\phi \in \mathcal{V}_{f}$ be $I_{f}$-invariant (i.e., $\eta^{*} \circ \phi \circ \sigma^{-1}=\phi$ for all $(\sigma, \eta) \in I_{f}$ ). Let $(\sigma, \eta)$ be as in the above theorem. Then

$$
-\phi(x)=\eta_{*} \phi(x)=\eta_{*} \phi\left(\sigma^{-1}(x)\right)=\phi(x) .
$$

Thus, $\phi(x)=0$ for any $x \in M$, and $f$ is hypercritical. 
CoROllaRy 4.2. The projection $p_{n, k}: V_{k}\left(\mathbf{F}^{n}\right) \rightarrow G_{k}\left(\mathbf{F}^{n}\right)(\mathbf{F}=\mathbf{R}, \mathbf{C}$, or $\mathbf{H})$ of the Stiefel manifold of orthonormal $k$-frames of $\mathbf{F}^{n}$ onto the Grassmann of $k$-planes of $\mathbf{F}^{n}$ is hypercritical, if $V_{k}\left(\mathbf{F}^{n}\right)$ and $G_{k}\left(\mathbf{F}^{n}\right)$ are given their standard Riemannian metrics.

Proof. Let $\pi$ be a $k$-plane in $\mathbf{F}^{n}$, and let $A: \mathbf{F}^{n} \rightarrow \mathbf{F}^{n}$ be reflection in the plane $\pi\left(A|\pi=I, A| \pi^{\perp}=-I\right)$. Then $A$ induces isometries

$$
A_{1}: V_{k}\left(\mathrm{~F}^{n}\right) \rightarrow V_{k}\left(\mathrm{~F}^{n}\right) \text { and } A_{2}: G_{k}\left(\mathrm{~F}^{n}\right) \rightarrow G_{k}\left(\mathrm{~F}^{n}\right)
$$

Indeed, $A_{2}$ is the geodesic symmetry of $G_{k}\left(\mathscr{F}^{n}\right)$ at $\pi$. Clearly $\left(A_{1}, A_{2}\right) \in I_{p_{k, n}}$. Moreover, $A_{1}$ leaves $p_{n, k}^{-1}(\pi)$ pointwise fixed. Thus, Theorem 4.1 applies.

COROLlaRy 4.3. Let $(M, g)$ and $\left(N, g^{\prime}\right)$ be Lie groups with bi-invariant metrics. If $f:(M, g) \rightarrow\left(N, g^{\prime}\right)$ is a homomorphism, then $f$ is hypercritical.

Proof. The geodesic symmetry $\sigma_{x}$ of $(M, g)$ at $x \in M$ is given by $\sigma_{x}\left(x^{\prime}\right)=$ $x x^{\prime-1} x$ (see [M, p. 112]). Let $\eta_{y}$ be the geodesic symmetry of $\left(N, g^{\prime}\right)$ at $y=f(x)$. Then

$$
\left(f \circ \sigma_{x}\right)\left(x^{\prime}\right)=f\left(x x^{\prime-1} x\right)=f(x) f\left(x^{\prime}\right)^{-1} f(x)=\left(\eta_{y} \circ f\right)\left(x^{\prime}\right) .
$$

Thus, $\left(\sigma_{x}, \eta_{y}\right) \in I_{f}$, and Theorem 4.1 applies.

THEOREM 4.4. Let $G$ be a connected Lie subgroup of the group of isometries of a Riemannian manifold $\left(N, g^{\prime}\right)$, and suppose $(M, g)$ is an orbit of $G(g=$ $\left.g^{\prime} \mid M\right)$. Assume that there is no nonzero $G$-invariant vector field on $M$, and no nonzero normal vector (to $M$ at some $x \in M$ ) that is fixed by the isotropy subgroup $G_{x}$. Then the inclusion $f:(M, g) \rightarrow\left(N, g^{\prime}\right)$ is hypercritical.

Proof. Certainly the diagonal $\Delta G \subset I_{f}$. If $\phi \in \mathfrak{V}_{f}$ is $I_{f}$ invariant, then $\phi^{T}$ (the component tangent to $M$ ) is a $G$-invariant vector field on $M$, while $\phi^{\perp}(x)$ is $G_{x}$-invariant. Thus, $\phi=\phi^{T}+\phi^{\perp}=0$.

COROLlaRy 4.5. The Veronese surface in $S^{4}$ is a hypercritical imbedding.

Proof. The Veronese surface $\left(\cong P_{2}(R)\right)$ has no nonzero vector field. Moreover, the Veronese surface is realized as a singular orbit of a group $(\simeq \mathrm{SO}(3))$ of isometries on $S^{4}$ (see [H2]). As there are no neighboring orbits of the same type, there can be no normal vectors invariant under the isotropy group. Indeed a computation reveals that if an element of the isotropy group rotates the tangent plane to the Veronese surface by $\theta$, then it rotates the normal plane by $2 \theta$.

THEOREM 4.6. Let $f:(M, g) \rightarrow\left(N, g^{\prime}\right)$ be a principal fiber bundle with group $H$ that acts on $M$ by isometries. Then $f$ is hypercritical iff there is no nonzero $I_{f}(N)$-invariant vector field on $N$. 
Proof. For $\sigma \in H$ we have $\left(\sigma, 1_{N}\right) \in I_{f}$. Hence, for $\phi \in \mathcal{V}_{f}$ with $\phi I_{f}$-invariant, we have

$$
\phi\left(\sigma^{-1}(x)\right)=\left(1_{N^{*}} \circ \phi \circ \sigma^{-1}\right)(x)=\phi(x) .
$$

Thus, there is a well-defined vector field $Y$ on $N$, given by $Y(f(x))=\phi(x)$. If $y=f(x)$ and $\eta \in I_{f}(N)$, say $(\sigma, \eta) \in I_{f}$, then

$$
\begin{aligned}
\left(\eta_{*} Y\right)(\eta y) & =\eta_{*}(Y(y))=\eta_{*}(\phi(x))=\phi(\sigma(x)) \\
& =Y(f(\sigma(x)))=Y(\eta(f(x)))=Y(\eta y) .
\end{aligned}
$$

Thus, $Y$ is $I_{f}(N)$-invariant. Conversely, if $Y$ is a $I_{f}(N)$-invariant vector field on $N$, then it is easy to verify that $\phi \in \mathscr{V}_{f}$, defined by $\phi(x)=Y(f(x))$, is $I_{f}$-invariant. Thus, the existence of a nonzero $I_{f}$-invariant $\phi \in \mathscr{V}_{f}$ is equivalent to the existence of a nonzero $I_{f}(N)$-invariant vector field on $N$.

We now consider a number of instructive, specific examples.

EXAMPLE 4.7. Let $E^{n}$ be Euclidean space and let $\Gamma$ and $\Gamma^{\prime}$ be subgroups of isometries of $E^{n}$ generated by $n$ independent translations. Let $f_{0}: E^{n} \rightarrow E^{n}$ be a linear transformation such that $f_{0}(\Gamma) \subset \Gamma^{\prime}$. Then $f_{0}$ induces a hypercritical map $f: E^{n} / \Gamma \rightarrow E^{n} / \Gamma^{\prime}$ of flat tori. This is just a special case of Corollary 4.3, but it serves to illustrate that critical maps need not be Riemannian in the sense of 3.12, even up to homothety.

The next examples will require a few facts on the geometry of left-invariant metrics on $S^{3}$, the group of unit quaternions. Let $X_{1}, X_{2}, X_{3}$ be left-invariant vector fields on $S^{3}$ such that $\left[X_{i}, X_{j}\right]=\varepsilon_{i j k} X_{k}$. We call such a frame field standard. Any two standard frame fields are related by an element of $\mathrm{SO}(3)$. Let $\theta_{1}, \theta_{2}, \theta_{3}$ be the dual one-forms of $X_{1}, X_{2}, X_{3}$. Consider the left-invariant metric $\sum a_{i} \theta_{i}^{2}, a_{i} \in \mathbf{R}^{+}$. Note that any left-invariant metric is obtained this way from some standard frame. Using the structural equations, we eventually find that the matrix of the Ricci curvature tensor, relative to the frame $Y_{i} \equiv \sqrt{a_{i}} X_{i}$, is diagonal with

$$
R_{11}=a_{1}\left(1-\frac{1}{2}\left(a_{2} a_{3}^{-1}+a_{3} a_{2}^{-1}\right)\right)+\frac{1}{2} a_{2} a_{3} a_{1}^{-1},
$$

etc. The case where $a_{1}=a_{2}=a_{3}=1$ yields a metric of constant sectional curvature $\frac{1}{4}$. The map $\left(a_{1}, a_{2}, a_{3}\right) \mapsto\left(R_{11}, R_{22}, R_{33}\right)$ has a nonsingular Jacobian matrix at $\left(a_{1}, a_{2}, a_{3}\right)=(1,1,1)$. Let us say that a left-invariant metric is Ricci-generic if its Ricci-tensor has distinct principal values relative to that metric. The above nonsingularity proves that the Ricci-generic metrics form the complement of a proper analytic set, and this complement is open and dense. The Christoffel symbols of $\sum a_{i} \theta_{i}^{2}$, relative to the frame $Y_{i}$ $\equiv \sqrt{a_{i}} X_{i}$, are given by

$$
\Gamma_{j k}^{i}=-\frac{1}{2} \varepsilon_{i j k}\left(a_{i}^{-1} a_{j} a_{k}+a_{k}^{-1} a_{i} a_{j}-a_{j}^{-1} a_{k} a_{i}\right) .
$$


Although $\nabla_{Y_{i}} Y_{i}=0(i=1,2,3)$, setting $Y=Y_{1}+Y_{2}$, we find

$$
\nabla_{Y} Y=a_{3}\left(a_{2} a_{1}^{-1}-a_{1} a_{2}^{-1}\right) Y_{3}
$$

which is nonzero if $a_{1} \neq a_{2}$ (i.e., $Y$ does not have geodesic integral curves).

EXAMPLE 4.8. Let $g=\sum a_{i} \theta_{i}^{2}$ be a left-invariant metric on $S^{3}$ with $a_{1} \neq a_{2}$. Let $H$ be the one-parameter subgroup of $S^{3}$ tangent to the left-invariant vector field $Y=Y_{1}+Y_{2}$ above. Let $g^{\prime}$ be a $S^{3}$-invariant metric on the quotient space $S^{3} / H \cong S^{2}$ ( $g^{\prime}$ is necessarily of constant curvature). The quotient map $f:\left(S^{3}, g\right) \rightarrow\left(S^{2}, g^{\prime}\right)$ is not hypercritical, because we have shown above that $H$ is not a geodesic (see also Theorem 3.11). Further analysis reveals that $f$ is not critical either. Thus, although $I_{f}\left(S^{3}\right)$ acts transitively on $S^{3}$, and $S^{2}$ admits no nonzero $I_{f}\left(S^{2}\right)$-invariant vector field, we still have an $I_{f}$-invariant vector field along $f$ which is not an infinitesimal isodeformation (see 3.8 and 3.10).

EXAmple 4.9. Let $X_{1}, X_{2}, X_{3}$ be a standard frame on $S^{3}$ with dual forms $\theta_{1}, \theta_{2}, \theta_{3}$. Let $g=\Sigma a_{i} \theta_{i}^{2}$ and $g^{\prime}=\Sigma a_{i}^{\prime} \theta_{i}^{2}$. Then the identity map $f:\left(S^{3}, g\right) \rightarrow$ $\left(S^{3}, g^{\prime}\right)$ is hypercritical. Indeed, let $A_{1}$ be the Lie algebra automorphism given by $A_{1}\left(X_{1}\right)=X_{1}, A_{1}\left(X_{2}\right)=-X_{2}, A_{1}\left(X_{3}\right)=-X_{3}$. Then $A_{1}$ induces an automorphism $\sigma_{1}: S^{3} \rightarrow S^{3}$ which is an isometry of $g$ and $g^{\prime}$, since $\sigma_{1 \cdot} \cdot\left(X_{1}\right)=X_{1}$, $\sigma_{1 *}\left(X_{2}\right)=-X_{2}, \sigma_{1^{*}}\left(X_{3}\right)=-X_{3}$. Similarly, we can define $\sigma_{2}$ and $\sigma_{3}$. Now $\left(\sigma_{i}, \sigma_{i}\right) \in I_{f}$, but there is no left-invariant nonzero vector field along $f$ (i.e. vector field on $S^{3}$ ) which is invariant under the operations $\phi \mapsto \sigma_{i^{*}} \circ \phi \circ \sigma_{i}^{-1}$ for $i=1,2,3$. Thus, $f$ is hypercritical.

EXAMPLE 4.10. Let $g$ and $g^{\prime}$ be left-invariant, Ricci-generic metrics on $S^{3}$ with no common principal Ricci directions. (This is possible by the remarks before 4.8.) Then the identity map $f:\left(S^{3}, g\right) \rightarrow\left(S^{3}, g^{\prime}\right)$ is not critical. If $\tau$ is a mutual isometry of $g$ and $g^{\prime}$ that fixes $e \in S^{3}$, then $\tau$ must preserve the principal Ricci directions of both $g$ and $g^{\prime}$ at $e$. By construction, $\tau_{* e}$ must be the identity, whence $\tau$ is the identity. Thus, $I_{f}$ is just the group $\{(\sigma, \sigma): \sigma$ is a left translation $\}$, and we see that any left-invariant vector field is $I_{f}$-invariant. Since the isotropy subgroups of $I_{g}$ or $I_{g^{\prime}}$ are discrete (of order 4), the Killing vector fields of $g$ or $g^{\prime}$ are precisely the right-invariant vector fields (of these, only the 0 field is also left-invariant). Thus, any nonzero left-invariant vector field will serve as an $I_{f}$-invariant vector field which is not an infinitesimal isodeformation.

As a corollary to Example 4.9, we have that for any left-invariant metric $g$ on $S^{3}$, the identity $f:\left(S^{3}, g\right) \rightarrow\left(S^{3}, g\right)$ is hypercritical. In this regard, $S^{3}$ (i.e., SU(2)) is probably the exception, rather than the rule. Consider:

EXAMPLE 4.11. Here we will outline a proof that there is a left-invariant metric $g$ on $\mathrm{SU}(3)$ such that the identity map $f:(\mathrm{SU}(3), g) \rightarrow(\mathrm{SU}(3), g)$ is not critical. Indeed, we will show that there is a left-invariant metric on SU(3) such that the isotropy subgroup of the full group of isometries is trivial. Then 
$f$ could not be critical, because of the same argument used in 4.10. Let $X_{1}, \ldots, X_{8}$ be a basis for the Lie algebra su(3) of SU(3). We call $X_{1}, \ldots, X_{8}$ a regular basis if there is no nontrivial automorphism of su(3) of the form $X_{i} \rightarrow \pm X_{i}$. Let $S$ denote the space of all inner products on su(3). If $g \in S$ and $R(g)$ denotes the Ricci tensor of the left-invariant metric determined by $g$, we define a map $\Omega: S \rightarrow L($ su (3)) (三 linear transformations of su(3)) by $g(\Omega(g) v, w)=R(g)(v, w)$, where we have made the identification $\operatorname{su}(3) \equiv$ $T_{e} \mathrm{SU}(3)$. The Ricci principal vectors and values of $g$ at $e$ are the eigenvectors and eigenvalues of $\Omega(g)$. Thus, $g$ determines a Ricci-generic metric if $\Omega(g)$ has distinct eigenvalues. We will presently reduce the problem to proving the following fact.

Assertion. There is a left-invariant Ricci-generic metric on SU(3) such that a regular basis of su(3) can be formed from Ricci principal vectors of the metric.

The reduction proceeds as follows. Let $g$ be the metric of the assertion. Since $g$ is Ricci-generic, any isometry $\tau$ of $g$ must preserve the Ricci directions of $g$ (i.e., $\tau_{*}\left(X_{i}\right)= \pm X_{i}$ ). In particular, $\tau_{*}$ maps left-invariant fields to left-invariant fields, and $\tau_{*}$ preserves Lie brackets of vector fields, as does the derivative of any diffeomorphism. If there is an isometry $\tau$ of $g$ that is not a left-translation, we may assume that $\tau$ fixes $e$. By the above remarks, $\tau_{* e}$ is an automorphism of $\operatorname{su}(3) \equiv T_{e} \mathrm{SU}(3)$, but this contradicts the regularity of the basis of Ricci-principal directions of $g$, unless $\tau_{*}$ is the identity or equivalently $\tau$ is the identity. The isotropy subgroup of $I_{g}$ is then trivial, as required.

We give a sketch of the proof of the assertion. First observe that the assertion can be restated in the following equivalent form: There is a metric $g$ such that $\Omega(g)$ commutes with no nontrivial involutive automorphism of su(3). Within the 8-dimensional space of all automorphisms of su(3), there are (up to conjugacy) just two types of nontrivial involutive automorphisms. Referring to [H1, p. 348], they are $\theta_{1}(X)=\bar{X}$ and $\theta_{2}(X)=I_{p, q} X I_{p, q}(p+q=$ $3 ; p, q \neq 0$ ). Now, the fixed algebra of $\theta_{1}$ is 3-dimensional, while that of $\theta_{2}$ is 4-dimensional. Hence, the space of all symmetric (relative to the Killing form) linear transformations commuting with $\theta_{1}$ is

$$
\frac{1}{2} 3(3+1)+\frac{1}{2} 5(5+1)=21 \text {-dimensional, }
$$

while for $\theta_{2}$ the corresponding space is $4(4+1)=20$-dimensional. Thus, the space of all symmetric transformations commuting with some conjugate of $\theta_{1}$ is (at most) 29-dimensional, and the corresponding space for $\boldsymbol{\theta}_{2}$ is 28-dimensional. Unfortunately, $\Omega(g)$ is not necessarily symmetric relative to $g_{0} \equiv$ - Killing form of su(3). Nevertheless, the differential at $g_{0}$ of $\Omega: S \rightarrow L(\operatorname{su}(3)$ ) has trivial kernel, and has image equal to the space $S^{\prime}$ of all symmetric 
(relative to $g_{0}$ ) linear transformations. This follows from a substantial calculation in which a $36 \times 36$ matrix is shown to be nonsingular. Now, there is an open and dense subset $S_{0}^{\prime} \subset S^{\prime}$ such that $A \in S_{0}^{\prime}$ implies $A$ has distinct eigenvalues and $A$ does not commute with any nontrivial involutive automorphism of su(3). For $A \in S_{0}^{\prime}$, let $g=d \Omega\left(g_{0}\right)^{-1}(A)$. Then, for all sufficiently small $\varepsilon>0, \Omega\left(g_{0}+\varepsilon g\right)=\frac{1}{4} I+\varepsilon A+o\left(\varepsilon^{2}\right)$ will have distinct eigenvalues and will not commute with any nontrivial involutive automorphism of su(3). As a consequence, we see that the space of all metrics satisfying the above assertion is the open and dense complement of a proper analytic set.

5. A characterization of hypercritical maps. The hypercritical maps $f:(M, g)$ $\rightarrow\left(N, g^{\prime}\right)$, for which $I_{f}(M)$ acts transitively on $M$, are the most suitable to characterize. We restrict ourselves to that case. Let $G=I_{f}(M), G^{\prime}=I_{f}(N)$, and $G^{\prime \prime}=\left\{\eta \mid f(M): \eta \in G^{\prime}\right\}$. Let $K$ be the isotropy subgroup of $G$ at a point $x \in M$, and let $H^{\prime \prime}$ be the isotropy subgroup of $G^{\prime \prime}$ at $y=f(x)$. We have a map $\psi: G \rightarrow G^{\prime \prime}$ given by $\psi(\sigma)(f(p))=f(\sigma(p))$. Let $H=\psi^{-1}\left(H^{\prime \prime}\right)$. Clearly $G \supset H \supset K$. There are maps $G \rightarrow M$ and $G \rightarrow f(M)$ given by $\sigma \mapsto \sigma(x)$ and $\sigma \mapsto \psi(\sigma)(y)=f(\sigma(x))$. These induce the vertical maps in the commutative diagram:

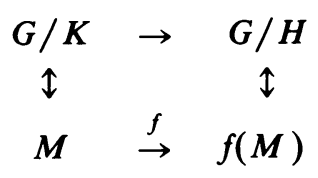

Let $\nu: \bar{V} \rightarrow f(M)$ be the normal bundle of $f(M) \subset N$. We have the restriction homomorphism $\psi^{\prime}: G^{\prime} \rightarrow G^{\prime \prime}$. Let $V$ be $\bar{V}_{y}=$ normal space to $f(M)$ at $y$. Setting $H^{\prime}=\psi^{\prime-1}\left(H^{\prime \prime}\right)$, we have the slice representation $\alpha: H^{\prime} \rightarrow O(V)$. Now, identify $\nu: \bar{V} \rightarrow f(M)$ with the vector bundle associated to the principal fiber bundle $G^{\prime} \rightarrow G^{\prime} / H^{\prime}$ via the representation $\alpha$. Denote this associated bundle by $G^{\prime} \times{ }_{\alpha} V \rightarrow G^{\prime} / H^{\prime}$. The identification of $\bar{V}$ with $G^{\prime} \times{ }_{\alpha} V$ is easily seen to be $G^{\prime}$-equivariant. Now, we can imbed $G^{\prime} \times{ }_{\alpha} V$ onto an open $\varepsilon$-tubular neighborhood $U$ of $f(M)$ in a $G^{\prime}$-equivariant fashion; so that we have the commutative diagram:

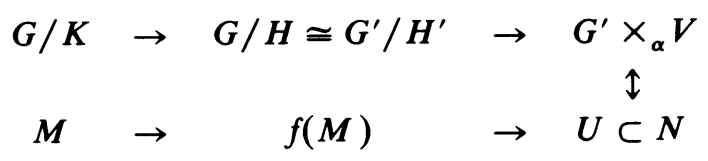

Set $K^{\prime \prime}=\psi(K) \subset H^{\prime \prime} \subset G^{\prime \prime}$ and $K^{\prime}=\psi^{\prime-1}\left(K^{\prime \prime}\right) \subset H^{\prime} \subset G^{\prime}$.

THEOREM 5.1. If $f:(M, g) \rightarrow\left(N, g^{\prime}\right)$ is hypercritical, then the representations $\left(\alpha \mid K^{\prime}\right): K^{\prime} \rightarrow O(V)$ and Ad: $K \rightarrow \operatorname{Hom}(\mathcal{S} / \mathcal{H C}, \mathcal{S} / \mathcal{H})$ each have no nonzero fixed vector. 
Proof. If $v \in V$ is fixed by all $\alpha(\tau), \tau \in K^{\prime}$, then define $\phi \in \mathcal{V}_{f}$ by $\phi(\sigma(x))=\eta_{*}(v)$ where $(\sigma, \eta) \in I_{f}$. This is well defined, because if $\left(\eta^{\prime}, \sigma^{\prime}\right) \in I_{f}$ and $\sigma^{\prime}(x)=\sigma(x)$, then we have $\sigma^{-1} \circ \sigma^{\prime} \in K$, from which we have $\eta^{-1} \circ \eta^{\prime} \in$ $K^{\prime}$. Hence,

$$
v=\alpha\left(\eta^{-1} \circ \eta^{\prime}\right)(v)=\eta_{*}^{-1} \circ \eta_{*}^{\prime}(v)
$$

or $\eta_{*}(v)=\eta_{*}^{\prime}(v)$. Now $\phi$ is also $I_{f}$-invariant. Indeed, let $x^{\prime}=\sigma(x)$ be an arbitrary point of $M$ where $\sigma \in I_{f}(M)$ with $(\sigma, \eta) \in I_{f}$. Then, for any $\left(\eta_{0}, \sigma_{0}\right)$ $\in I_{f}$, we have

$$
\begin{aligned}
\eta_{0 *} \phi\left(\sigma_{0}^{-1}\left(x^{\prime}\right)\right) & =\eta_{0 *} \phi\left(\sigma_{0}^{-1} \circ \sigma(x)\right) \\
& =\eta_{0 *} \circ\left(\eta_{0}^{-1} \circ \eta\right)_{*}(\phi(x))=\eta_{*}(v) \\
& =\phi(\sigma(x))=\phi\left(x^{\prime}\right) .
\end{aligned}
$$

Since $f$ is hypercritical, we have $\phi=0$, or $v=0$, as required.

Now let $w \in \mathcal{G} / \mathcal{H}$ be fixed by the adjoint representation Ad: $K \rightarrow \mathcal{G} / \mathcal{H}$. Under the isomorphism in diagram (1), the tangent space of $f(M)$ at $y$ can be identified with $\mathcal{G} / \mathcal{H}$. Let $Y \in T_{y} f(M)$ correspond to $w$, and define $\phi \in \mathcal{V}_{f}$ by the formula $\phi(\sigma(x))=\eta_{*}(Y)$ for $(\sigma, \eta) \in I_{f}$. We show that $\phi$ is well defined. Let $\left(\sigma^{\prime}, \eta^{\prime}\right) \in I_{f}$ with $\sigma^{\prime}(x)=\sigma(x)$, then $\sigma^{\prime} \circ \sigma^{-1} \in K$. Using diagram (1), we see that the $\operatorname{Ad}(K)$ invariance of $w$ implies that

$$
\left[\left(\eta^{\prime} \mid f(M)\right) \circ(\eta \mid f(M))^{-1}\right]_{*}(Y)=Y
$$

or $\eta_{*}^{\prime}(Y)=\eta_{*}(Y)$, and so $\phi$ is well defined. The proof of the $I_{f}$ invariance of $\phi$ is the same as before, only replace $v$ by $Y$. Since $f$ is hypercritical, $\phi=0$, whence $w=0$.

We now formulate a converse (of sorts) to Theorem 5.1. Let $G, G^{\prime}, G^{\prime \prime}$ be compact Lie groups, and suppose we have epimorphisms $\psi: G \rightarrow G^{\prime \prime}$ and $\psi^{\prime}$ : $G^{\prime} \rightarrow G^{\prime \prime}$. Let $K$ be a closed subgroup of $G$ such that $G / K$ is connected, and suppose that $H^{\prime \prime}$ is a closed subgroup of $G^{\prime \prime}$ such that $H \equiv \psi^{-1}\left(H^{\prime \prime}\right) \supset K$, and Ad: $K \rightarrow \operatorname{Hom}(\mathcal{G} / \mathcal{H}, \mathcal{G} / \mathcal{H})$ has nonzero fixed vectors. Let $\boldsymbol{H}^{\prime}=$ $\left(\psi^{\prime}\right)^{-1}\left(H^{\prime \prime}\right) \subset G^{\prime}$, and let $\alpha: H^{\prime} \rightarrow \operatorname{Hom}(V, V)$ be a representation such that (for $K^{\prime \prime}=\psi(K)$ and $K^{\prime}=\psi^{\prime-1}\left(K^{\prime \prime}\right)$ ) $\alpha \mid K^{\prime}$ fixes no nonzero $v \in V$. Let $g$ be a $G$-invariant metric on $G / K$, and let $g^{\prime}$ be a $G^{\prime}$-invariant metric on $G \times{ }_{\alpha} V$. Assume that the zero section $s: G^{\prime} / H^{\prime} \rightarrow G \times{ }_{\alpha} V$ meets the fibers orthogonally. This can always be accomplished by averaging (over the compact group $G^{\prime}$ ) any non- $G^{\prime}$-invariant metric for which $s$ meets the fibers orthogonally.

THEOREM 5.2. With notation as above, we have that the following composition is hypercritical:

$$
f:(G / K, g) \rightarrow G / H \cong G^{\prime \prime} / H^{\prime \prime} \cong G^{\prime} / H^{\prime} \stackrel{s}{\rightarrow}\left(G^{\prime} \times_{\alpha} V, g^{\prime}\right) .
$$


Proof. Observe that $I_{f}$ contains the subgroup of all pairs of left translations $\left(L_{g}, L_{g^{\prime}}\right)$ such that the induced left translations on $G^{\prime \prime} / H^{\prime \prime}$ agree. Note that $I_{f}$ may be considerably larger than this subgroup, which we call $J_{f}$. Suppose $\phi \in \mathcal{V}_{f}$ is $I_{f}$-invariant. Then $\phi$ is certainly $J_{f}$-invariant as well. The components of $\phi$, say $\phi^{T}$ and $\phi^{N}$ (in the directions tangent and normal to the image of $s$ ) are seen to be $J_{f}$-invariant. The assumptions on the representations Ad: $K \rightarrow \operatorname{Hom}(\mathcal{G} / \mathcal{H}, \mathcal{G} / \mathcal{H})$ and $\alpha: H^{\prime} \rightarrow \operatorname{Hom}(V, V)$ imply that $\phi^{T}=0$ and $\phi^{N}=0$, whence $\phi=0$.

6. Some open problems. 1. Prove or disprove that "critical" and "hypercritical" are equivalent notions.

2. Determine which of the minimal immersions of various isotropy irreducible homogeneous spaces into spheres, via eigenfunctions of the Laplacian, are critical (see [B1, p. 174]). Corollary 4.5 is a start.

3. As a follow-up on Example 4.11, prove or disprove that every compact, simply-connected, simple Lie group (of dimension $>3$ ) has a dense set of left-invariant metrics for which the isotropy subgroup of the full group of isometries is trivial. For such metrics, the identity map is not critical.

4. Find all critical maps $f:(M, g) \rightarrow\left(N, g^{\prime}\right)$ for low-dimensional $M$ and $N$, assuming that $I_{f}(M)$ acts transitively on $M$.

5. As critical maps $f:(M, g) \rightarrow\left(N, g^{\prime}\right)$ may be too numerous to suitably classify, it is both natural and desirable to assume that $(M, g)$ (and possibly $\left.\left(N, g^{\prime}\right)\right)$ is an isotropy irreducible homogeneous space (see [W]). Determine how far a classification, under this assumption, can be carried out.

6. Prove a slice theorem for the action of $\mathscr{D}(M) \times I_{g^{\prime}}$ on $\mathscr{N} \times C^{\infty}(M, N)$ $\times\left\{g^{\prime}\right\}$, and justify Definition 2.2. (It appears that this may follow fairly directly from a yet unpublished paper of Palais dealing with the existence of slices under more general circumstances.)

7. Let $\mathscr{F}_{k}$ be the set of functionals on $\mathfrak{T} \times C^{\infty}(M, N) \times\left\{g^{\prime}\right\}$ which are defined by integrating some "natural" density depending only on the $k$-jet of the map. Is there some $k$, such that if a map is critical for all functionals in $\mathscr{F}_{k}$, then it is critical?

8. Are there any apparently mild conditions on a holomorphic map between Kähler manifolds which insure that the map is critical? (For example, such a map is automatically harmonic.)

9. A map $f: M \rightarrow N$ can be regarded as a section of the product bundle $M \times N \rightarrow M$. This suggests that one should try to formulate a notion of a critical section of a (Riemannian) fiber bundle, and generalize the results here.

10. One can probably establish some theorems concerning the uniqueness (up to equivalence) of critical maps in a given homotopy class of mappings (e.g. see [E3] for some results in this direction). 


\section{BIBLIOGRAPHY}

[B1] M. Berger, Le spectre d'une variété riemannienne, Lecture Notes in Math., vol. 194, Springer-Verlag, Berlin, 1971.

[B2] M. Berger and D. Ebin, Some decompositions of symmetric tensors on a Riemannian manifold, J. Differential Geometry 3 (1969), 379-392.

[B3] D. Bleecker, Critical Riemannian manifolds, J. Differential Geometry (to appear).

[E1] D. Ebin, The manifold of Riemannian metrics, Proc. Sympos. Pure Math., vol. 15, Part 2, Amer. Math. Soc., Providence, R. I., 1970, pp. 11-40.

[E2] J. Eells and J. Sampson, Harmonic mappings of Riemannian manifolds, Amer. J. Math. 86 (1964), 109-160.

[E3] R. Escobales, Riemannian submersions with totally geodesic fibers, J. Differential Geometry 10 (1975), 253-276.

[H1] S. Helgason, Differential geometry and symmetric spaces, Academic Press, New York, 1962.

[H2] W. Y. Hsiang and H. B. Lawson, Minimal submanifolds of low cohomogeneity, J. Differential Geometry 5 (1971), 1-38.

[M] J. Milnor, Morse theory, Princeton Univ. Press, Princeton, N. J., 1963.

[O] B. O'Neill, The fundamental equations of a submersion, Michigan Math. J. 13 (1966), 459-469.

[W] J. A. Wolf, The geometry and structure of isotropy irreducible homogeneous spaces, Acta Math. 120 (1968) 50-148.

Department of Mathematics, University of Hawail at Manoa, 2565 The Mall, HoNOlulu, HaWAII 96822 